\title{
Experimental investigation on pore size distribution and drying kinetics during lyophilization of sugar solutions
}

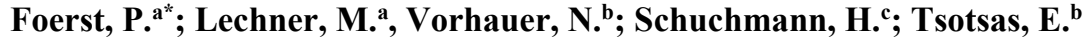

${ }^{a}$ Chair of Process Systems Engineering. Technical University of Munich, Freising, Germany

${ }^{b}$ Chair of Thermal Process Engineering. Otto-von-Guericke University Magdeburg, Germany

c Wilhelm Büchner Hochschule, Pfungstadt, Germany

*E-mail of the corresponding author: petra.foerst@tum.de

\begin{abstract}
The pore structure is a decisive factor for the process efficiency and product quality of freeze dried products. In this work the two-dimensional ice crystal structure was investigated for maltodextrin solutions with different concentrations by a freeze drying microscope. The resulting drying kinetics was investigated for different pore structures. Additionally the threedimensional pore structure of the freeze dried samples was measured by $\mu$ computed tomography and the pore size distribution was quantified by image analysis techniques. The two- and three-dimensional pore size distributions were compared and linked to the drying kinetics.
\end{abstract}

Keywords: pore size distribution; freeze drying; maltodextrin solution; freeze drying microscope 


\section{Introduction}

Lyophilisation or freeze drying is commonly applied to stabilize (bio-)pharmaceutical substances and high value foods for long-term storage. During drying, the thickness of the dried layer is steadily increasing. Therefore, the mass transfer resistance becomes the dominating factor causing very long process times [1]. The mass transfer resistance is directly linked to the pore structure of the frozen solution. The pore structure is also important for the freeze drying of particulate matter as it determines the intra-particle mass transfer resistance and process efficiency. Furthermore, collapse phenomena are directly linked to the pore structure as the pore structure determines the maximum product temperature. The pore structure is determined by the freezing process prior to sublimation and is depending on cooling rate, nucleation temperature and solid content of the solution [2].

The pore structure is the decisive factor for the formation of the drying front of both particulate systems and frozen liquids. Therefore it is the aim of this work to investigate the influence of the process parameters cooling rate, annealing treatment and solid concentration on the pore size distribution of maltodextrin solutions as model systems.

Regarding the quantitative evaluation of pore size distribution it has to be distinguished between the evaluation of the frozen system and the dried system. In most cases it is assumed that no shrinkage occurs during freeze drying and the pore size of the frozen system is not different to the dried system. For frozen systems the ice crystal size distribution is mostly determined from sliced frozen samples by light microscopic techniques or Cryo-SEM [3]. In dried systems, mostly SEM and more recently $\mu$-computed tomography are used for the evaluation of pore structure of freeze dried products $[4,5]$. The aim of this work is to compare the two-dimensional ice crystal structure as observed in a freeze drying microscope with the three-dimensional pore structure measured by $\mu$ computed tomography $(\mu-\mathrm{CT})$ and to link the pore structure to the drying kinetics.

\section{Materials and Methods}

Maltodextrin solution (DE 10-14 from medesign I.C. GmbH) was used as model substance. Solutions of maltodextrin were prepared by using bi-distilled water with the following concentrations: $5 \%, 10 \%$ and $20 \%(\mathrm{w} / \mathrm{w})$.

\subsection{Freeze drying microscope}

The controlled cooling of the solutions was carried out with the cryostage FDCS196 (Linkam Instruments, Tadworth, UK) coupled to an Olympus BX51 polarized light microscope (Olympus Microscopy, Essex, UK) equipped with a Pixelink PL-A662 camera (Pixelink, Rochester, USA). A quantitiy of $10 \mu \mathrm{l}$ of the sample was loaded into a $15 \mathrm{~mm}$ 
diameter quartz glass crucible, which was placed on a silver block in the stage. The controlled temperature ranged between $-196^{\circ} \mathrm{C}$ to $125^{\circ} \mathrm{C}$. The sample was enclosed by a spacer to achieve a uniform thickness of $690 \mu \mathrm{m}$. Thus the sample forms a cylindrical disk with a diameter of $4.3 \mathrm{~mm}$ and a height of $690 \mu \mathrm{m}$. Temperature was measured using a Pt100 temperature sensor from Linkam Instruments (accuracy $\pm 0.1^{\circ} \mathrm{C}$ ). By calibrating the 20x magnification lens (LMPlan F1 20x/0.40) with a scale, the lengths and dimensions of the microscopic images were calculated using Linksys32 software.

\subsection{Freezing and drying experiments and microscopic image analysis}

After placing the liquid sample onto the quartz crucible, the stage was sealed and all samples were cooled to $-50^{\circ} \mathrm{C}$ at a defined cooling rate. Two different cooling rates were investigated: $0.5 \mathrm{~K} / \mathrm{min}$ and $20 \mathrm{~K} / \mathrm{min}$. Furthermore two annealing treatments were investigated: In Treatment I the sample was heated to $-10{ }^{\circ} \mathrm{C}$ for $90 \mathrm{~min}$ after freezing and cooled back to $-50{ }^{\circ} \mathrm{C}$. In treatment II the sample was heated to $-5{ }^{\circ} \mathrm{C}$ after freezing for $180 \mathrm{~min}$ and cooled back to $-50{ }^{\circ} \mathrm{C}$. After reaching $-50{ }^{\circ} \mathrm{C}$, pictures were taken to evaluate the two-dimensional ice-crystal size distribution of the frozen samples. The subsequent drying step was carried out with a shelf temperature of $-18{ }^{\circ} \mathrm{C}$ and a pressure of $10 \mathrm{~Pa}$ for all freezing protocols. During drying, a picture was taken every $10 \mathrm{~s}$. As soon as primary drying was finished which was determined by the disappearance of the drying front, the silver block was heated to $30{ }^{\circ} \mathrm{C}$ and the secondary drying was carried out overnight until a final water content of approx. $2 \%$ was achieved. The samples were then taken out of the cryostage and stored in a desiccator until further evaluation of the dry pore structure by $\mu$ CT.

For the evaluation of the ice crystal size, the software Linksys 32 was used. Three pictures of each sample were taken and 100 ice crystals were manually analysed per picture. The ice crystals were analysed by edging the ice crystals. From the ice crystal contour the equivalent diameter $x_{p m}$ of a sphere with equal area of projection was obtained.

\section{$2.3 \mu-C T$ measurements and image analysis}

The samples that were evaluated for ice crystal size and drying kinetics, were taken for the $\mu$-CT analysis as well. Three-dimensional X-ray $\mu$-CT scans were performed using the XCT1600 system from Nordson Matrix Technologies GmbH (Feldkirchen, Germany). The cylindrical freeze-dried cakes from the freeze drying microscope were cut with a razor knife to prepare cuboid pieces (length and width about $2 \mathrm{~mm}$ ) out of the center of the dried solids. The imaging system was adjusted to operate at a tube voltage of $60 \mathrm{kV}$ and current intensity of $20 \mu \mathrm{A}$. X-ray shadow images were acquired with a pixel resolution of $1 \mu \mathrm{m}$ and 2000 projections with an exposure time of $4487 \mathrm{~ms}$. The obtained images were reconstructed into a series of 2-D images using the CERA Xplorer software (Siemens 
Healthcare GmbH, Erlangen). The resulting 3-D images consisted of more than 2000 slices encoded in 16-bit precision.

Image processing and analysis were done with MAVI Fraunhofer ITWM software (Fraunhofer Institute for Industrial Mathematics, Kaiserslautern). Fig. 2 shows part of the image analysis steps performed with MAVI. First, the original data set was cropped into three cubic sub-volumes of 400x400x400 voxels equivalent to $400 \times 400 \times 400 \mu \mathrm{m}^{3}$ (Fig. 2a). Afterwards, a global threshold was defined to separate pores and solid. The global threshold was adjusted to the water content in the solutions; i.e. 0.8 for the $20 \%$ solution, 0.9 for the $10 \%$ solution and 0.95 for the $5 \%$ solution. After transformation of the greyscale into binary images (Fig. 2b), a Euclidean distance transformation was carried out, which created a distance map by assigning each background voxel a grey value based on the distance to the closest foreground voxel (Fig. 2c). Thereby, local minima were identified. To avoid oversegmentation of pore structure, a h-minima transformation was then performed with a global $\mathrm{h}=10$. This value was chosen by comparison of the results with the twodimensional ice-crystal structure (see section 3.1). Subsequently, the individual pores were segmented using the watershed segmentation method. All remaining regional minima served as water sources for the flooding. The lines where water from different basins met were identified as the watershed lines. Separated cells were produced from the logical difference between the watershed lines and the initial binary image (Fig. 2d). Finally, the object features tool of MAVI was used to obtain the volume of the individual pores. The equivalent pore diameter values were determined by calculating the diameters of spheres corresponding to the pore volumes. Therefore the equivalent diameter $\mathrm{x}_{\mathrm{v}}$ of a sphere with the same volume was determined.

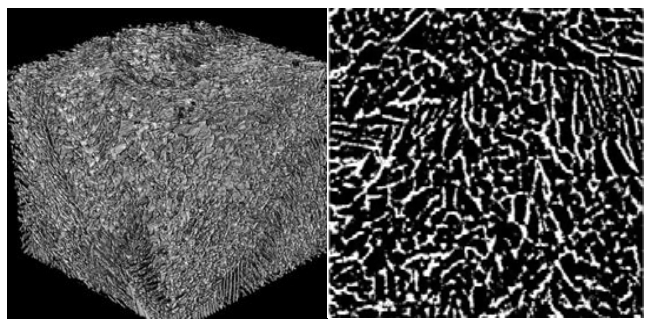

a) b)

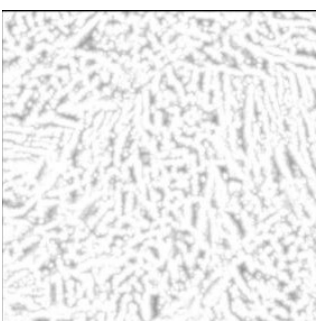

c)

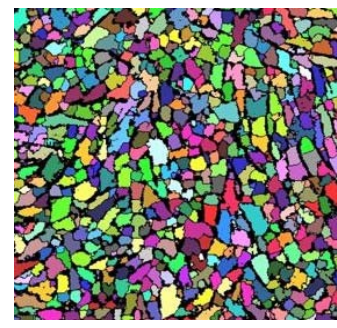

d)

Fig. 2. Illustration of all steps performed for the image analysis with MAVI: 3D image of cubic sub-volumes of 400x400x400 voxels (a), binarized image (c), distance map after Euclidean distance transform (d), watershed image (e)

\subsection{Evaluation of drying rate}

After keeping the sample at $-50^{\circ} \mathrm{C}$ for $15 \mathrm{~min}$ the pressure was decreased by a rotary vane pump to $10 \mathrm{~Pa}$. Then the samples were heated to $-18{ }^{\circ} \mathrm{C}$ at a rate of $3 \mathrm{~K} / \mathrm{min}$. Due to the cylindrical geometry of the sample, the drying proceeded in radial direction. As the dried part of the sample appears much darker than the frozen part, the radial drying front could be 
easily visualized. The distance between edge of the sample $\left(\mathrm{R}_{0}\right)$ and the drying front over time $r(t)$ was measured at three positions every minute. From the movement of the drying front $\Delta \mathrm{r}$ the drying rate was calculated.

$$
\dot{m}=\frac{\rho_{\text {ice }} 2 \pi r h \varepsilon \Delta r}{\Delta t}
$$

Here, $\rho_{\text {ice }}$ is the density of ice at the sublimation temperature, $r$ is the radius of the sublimation front, $\mathrm{h}$ the height of the sample $(690 \mu \mathrm{m}), \varepsilon$ the porosity and $\Delta \mathrm{r} / \Delta \mathrm{t}$ the change in sublimation front distance.

\section{Results}

\subsection{D ice crystal structure}

At first, the two-dimensional ice-crystal size distribution was evaluated for the different freezing protocols and solid concentrations. Fig. 3 shows the two-dimensional ice crystal size distribution for three different solid concentrations with and without annealing treatment.

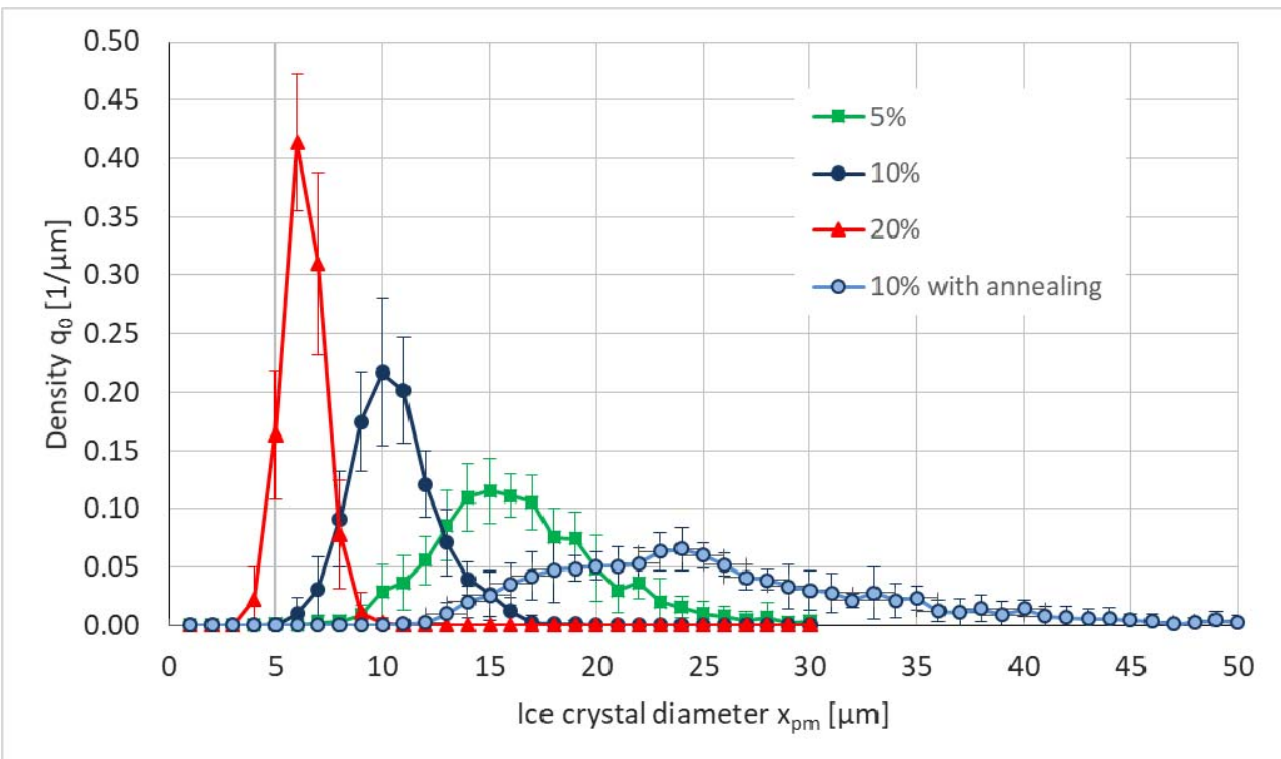

Fig. 3. Ice crystal diameter for frozen maltodextrin solutions at $-50^{\circ} \mathrm{C}$ for different solid contents. Freezing was carried out with a cooling rate of $20 \mathrm{~K} / \mathrm{min}$. For the $10 \%$ solution, the ice crystal size distribution for annealing treatment $\mathrm{II}\left(-5^{\circ} \mathrm{C} / 180 \mathrm{~min}\right)$ is also shown 
It was found that the freezing rate had no significant influence on ice crystal size

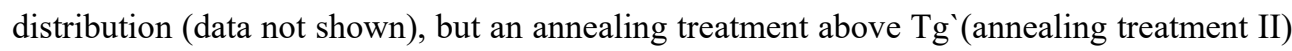
led to a much broader and coarser ice crystal size whereas the annealing treatment I had no influence on ice crystal size (data not shown). Next to the annealing treatment, the solid concentration had the largest impact on ice crystal size. Fig. 3 shows that the lowest solid concentration (5\%) leads to the largest ice crystal diameters and also to the broadest distribution.

\subsection{D pore structure}

The cylindrical disks prepared in the freeze drying microscope were further dried until about a water content of $2 \%$. From the dried samples, a $\mu$-CT image with a resolution of 1 $\mu \mathrm{m}$ was taken in order to evaluate the three-dimensional pore structure. As indicated in section 2.3, a h-minima transformation was performed to avoid oversegmentation. The $h$ value is therefore a free parameter. The parameter $h$ was chosen such that the modes of the $2 \mathrm{D}$ and $3 \mathrm{D}$ distribution coincide. The comparison between $2 \mathrm{D}$ ice crystal diameter $\mathrm{x}_{\mathrm{pm}}$ and 3D for the different freezing protocols and solid contents is shown in Fig. 4.

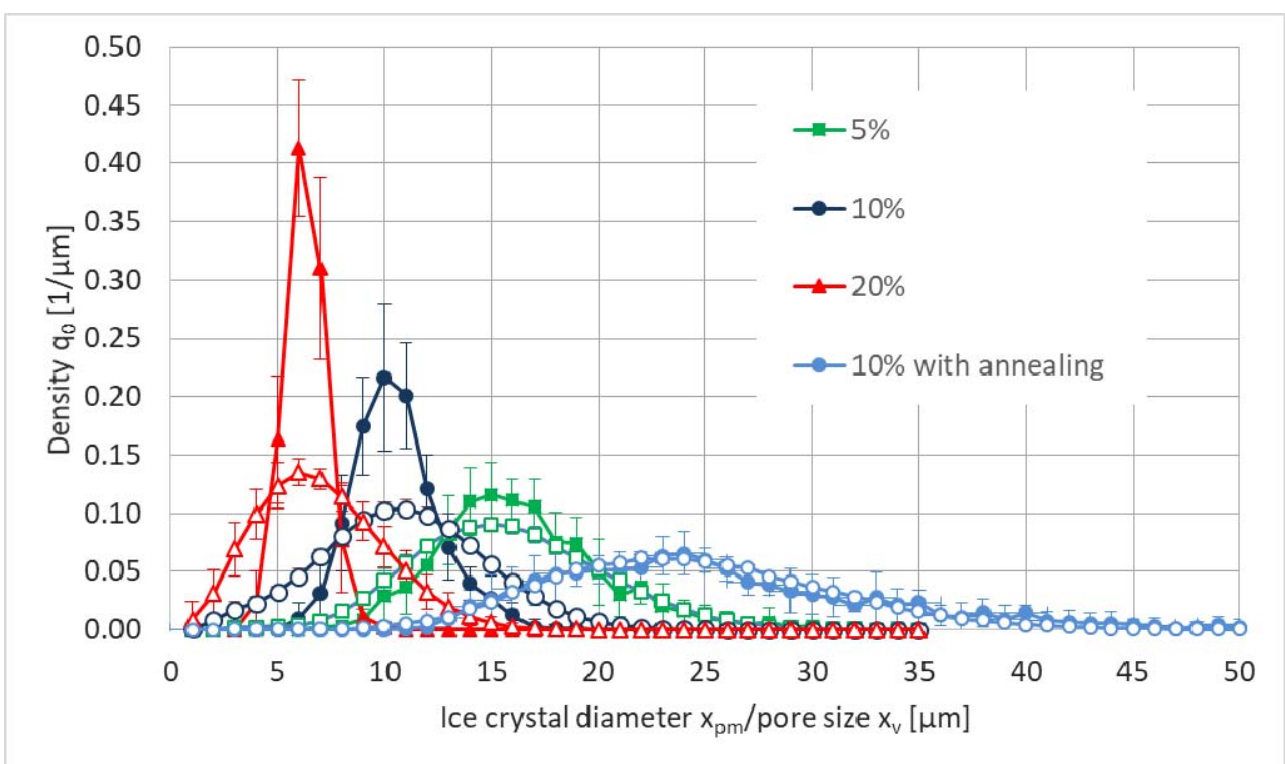

Fig. 4. Comparison between 2D and 3D Structure. Density distribution of ice crystal sizes (filled symbols) in comparison to pore sizes of dried cake (empty symbols) for different solid contents (freezing rate $20 \mathrm{~K} / \mathrm{min}$ ) and annealing treatment

Fig. 4 shows that the discrepancy between $2 \mathrm{D}$ ice crystal size and $3 \mathrm{D}$ pore size becomes smaller with increasing pore size. The reason could be that the resolution limit in the light microscope is higher than in the $\mu$-CT and therefore the larger sizes match better. As both 
measurements refer to different equivalent diameters, another reason could be that the shape of the pores is dependent on size and that small pores are less spherical. This issue has to be investigated in more detail in future. At last we were interested in the effect of pore structure on drying kinetics. For that we investigated the relationship between mean ice crystal size (modal value) and maximum drying rate as well as drying time. This correlation is shown in Fig. 5. An exponential relationship was found showing the high importance of pore size on drying kinetics.

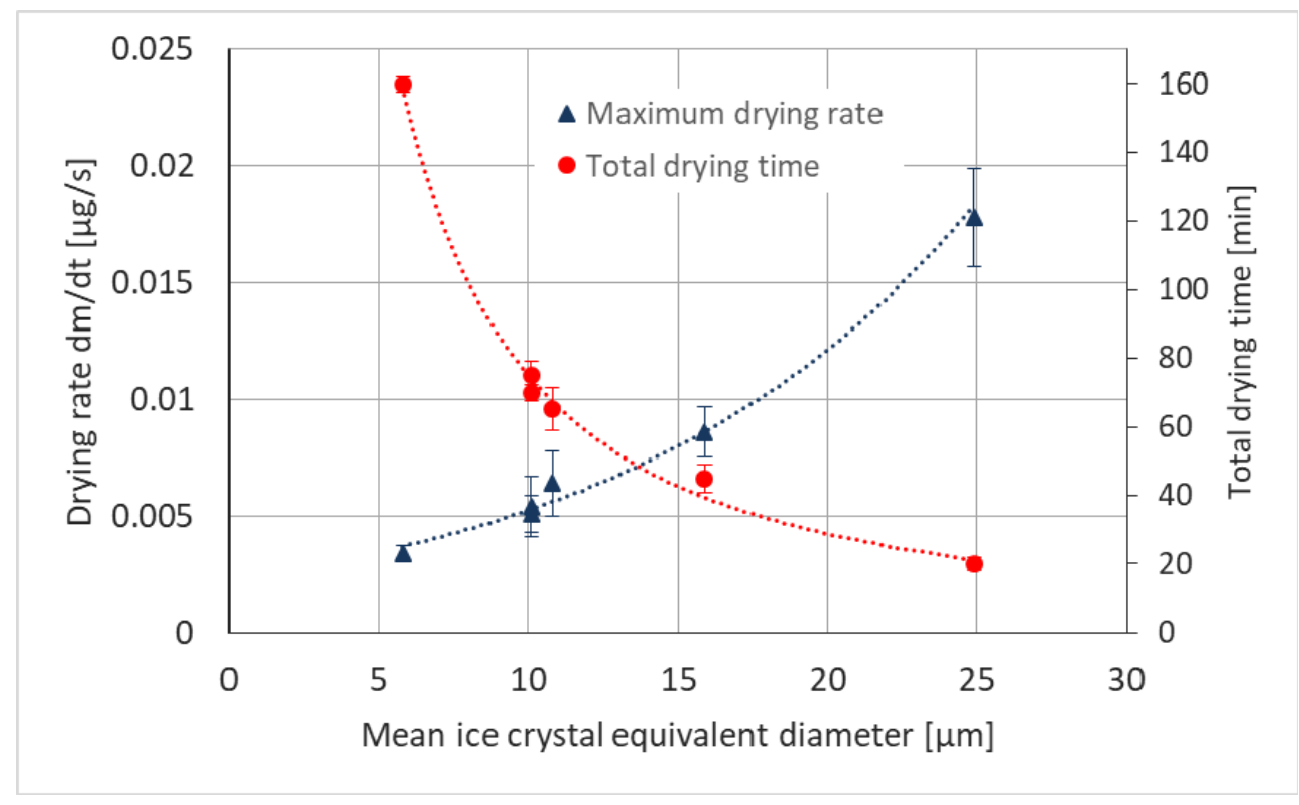

Fig. 5.: Correlation between mean ice crystal diameter and maximum drying rate and total drying

\section{Conclusions} time.

The work shows the high importance of pore size distribution for the freeze drying process. It was shown that it is possible to evaluate the three-dimensional pore structure with $\mu$ computed tomography and image analysis and that the pore size is strongly dependent on the solid concentration and on an annealing treatment prior to drying. This means that it is possible to design the pore structure to meet the requirements of the freeze drying process. The work shows the applicability of the $\mu$-CT measurement of pore size distribution and shows that for small pore sizes there is a discrepancy between two- and three-dimensional pore size. This relationship has to be investigated further. 


\section{Nomenclature}

$\begin{array}{llc}\mathrm{X}_{\mathrm{pm}} & \text { diameter of sphere with same mean area of projection } & \mathrm{m} \\ \mathrm{X}_{\mathrm{V}} & \text { diameter of sphere with same volumt } & \mathrm{m} \\ \mathrm{h} & \text { height } & \mathrm{m} \\ \mathrm{R} & \text { radius of sample } & \mathrm{m} \\ \mathrm{r} & \text { radius of sublimation front } & \mathrm{m} \\ \mathrm{q}_{0} & \text { density distribution } & \mu \mathrm{m}^{-1}\end{array}$

Greek letters

$\begin{array}{lll}\varepsilon & \text { porosity } & - \\ \rho & \text { density } & \mathrm{kgm}^{-3}\end{array}$

Subscripts

$\begin{array}{ll}\text { ice } & \text { ice } \\ 0 & \text { initial }\end{array}$

\section{References}

[1] Pliske. R.; Müller, U.; Kohlus, R. Untersuchungen zur Steigerung der Trocknungsgeschwindigkeit der Gefriertrocknung. Chemie Ingenieur Technik 2015, 87(7), 1006-1010

[2] Searles, J. A.; Carpenter, J.F.; Randolph, T.W. The ice nucleation temperature determines the primary drying rate of lyophilization for samples frozen on a temperature-controlled shelf. Journal of Pharmaceutical Sciences 2001, 90, 860-871

[3] Hottot, A.; Vessot, S.; Andrieu, J. A direct characterization method of the ice morphology. Relationship between mean crystals size and primary drying times of freeze-drying processes. Drying Technology 2004, 22(8), 2009-2021

[4] Pisano, R.; Barresi, A.A.; Capozzi, L.C: Novajra, G.; Oddone, I.; Vitale-Brovarone, C. Characterisation of the mass transfer of lyophilized products based on X-ray microcomputed images. Drying Technology 2017, 35(8), 933-938

[5] Mousavi, R.; Miri, T.; Cox, P. W.; Fryer, P. J. A Novel Technique for Ice Crystal Visualization in Frozen Solids Using X-Ray Micro-Computed Tomography. Journal of Food Science 2005, 70(7), 437-442. 